\title{
Variable Central Stars of Young Planetary Nebulae
}

\author{
G. Handler \\ Institut für Astronomie, Universität Wien, Austria
}

Three different kinds of variable stars can be found among central stars of Planetary Nebulae. Close binary central stars change their light level because of eclipses and due to the reflection effect; some hot variables have been identified as multiperiodic pulsators (e.g. see Ciardullo \& Bond 1996).

The interpretation of the behavior of the third class of variables is a more complicated problem. They have effective temperatures between 25000 and $50000 \mathrm{~K}$ and possess compact nebulae of high surface brightness. These stars show photometric and radial velocity variations with time scales of several hours, and no well-defined single periodicity seems to be present in these variations.

We (Handler et al. 1996, Méndez et al. 1996) organised one photometric multisite campaign for the central star of IC 418 (the prototype object), one simultaneous photometric and spectroscopic campaign for the same star and we carried out a survey for variability among these stars (Handler 1996).

From these data, we can rule out rotational modulation of surface features as the cause of the variability, and we conclude that most of these stars cannot be close binaries. We cannot yet exclude wind variability as a possible explanation. However, if wind variations are present, then the mechanism causing them is different from the one operating in massive $\mathrm{O}$ stars.

The most likely explanation for the variability of central stars of young Planetary Nebulae is pulsation. The time scales for radial fundamental mode pulsation (as inferred from model calculations) are in good agreement with the measured time scales of variability.

To prove or disregard the latter two hypotheses, we will carry out further multisite campaigns of a number of variable central stars. Less well studied objects will be observed through single or two-site runs. Pulsational model calculations will also be undertaken.

This research was partly supported by the Austrian Fonds zur Förderung der wissenschaftlichen Forschung under grant No. S-7309. The author acknowledges partial financial support by the Austrian Zentrum für Auslandsstudien and by the Österreichische Forschungs gemeinschaft as well.

\section{REFERENCES}

Ciardullo, R., Bond, H. E., 1996, AJ 111, 2332

Handler, G., 1996, in preparation

Handler, G., Méndez, R. H., Medupe, R., et al., 1996, A\&A, in press

Méndez, R. H., et al., 1996, in preparation 